\title{
VECTOR BUNDLES WITH NO SOUL
}

\author{
MURAD ÖZAYDIN AND GERARD WALSCHAP
}

(Communicated by Christopher Croke)

\begin{abstract}
We exhibit vector bundles over compact manifolds of nonnegative sectional curvature whose total spaces do not admit a complete metric of nonnegative sectional curvature.
\end{abstract}

According to the Cheeger-Gromoll theorem [1], any complete, noncompact Riemannian manifold with nonnegative sectional curvature $K \geq 0$ is diffeomorphic to the normal bundle of a compact, totally convex submanifold $S$, called a soul of $M$. In many respects, this structure theorem reduces the study of open manifolds with $K \geq 0$ to that of compact ones, since a soul, being totally geodesic, is necessarily nonnegatively curved. Conversely, any compact nonnegatively curved manifold $S$ can be realized as a soul, for instance, by taking $M$ to be an isometric product $S \times \mathbb{R}^{k}$. A natural question, then, is whether the total space $E$ of any vector bundle over a compact manifold with $K \geq 0$ admits a metric of nonnegative curvature. This is certainly true if $\operatorname{dim} E \leq 3$ [1]. In this note, we show it is no longer true in general when $\operatorname{dim} E \geq 4$, by observing that among all oriented plane bundles over the torus $T^{2}$, only the trivial one admits such a metric. These bundles are, up to equivalence, in 1-1 correspondence with $H^{2}\left(T^{2}\right)$ via their Euler class. A key fact here is that nonvanishing of the Euler class is actually a topological feature of the total space rather than of the bundle.

We wish to thank $\mathrm{H}$. Blaine Lawson for a helpful conversation regarding this paper.

Theorem. Let $E$ denote the total space of a rank $k$ vector bundle over a Bieberbach manifold $M^{n}$ (i.e., a compact manifold admitting a flat metric). Then the following statements are equivalent:

(1) E admits a complete metric of nonnegative curvature.

(2) E admits a complete flat metric.

(3) $E$ is diffeomorphic to the total space of a rank $k$ vector bundle over $M$ which admits a flat Riemannian connection.

(4) $E$ is diffeomorphic to $\mathbb{R}^{n} \times_{\pi_{1}(M)} \mathbb{R}^{k}$, where $\pi_{1}(M)$ acts on $\mathbb{R}^{n}$ by covering transformations and on $\mathbb{R}^{k}$ via some orthogonal representation.

Received by the editors April 14, 1992 and, in revised form, May 28, 1992.

1991 Mathematics Subject Classification. Primary 53C20. 
Proof. The equivalence of (3) and (4) is due to Milnor [3]. Since (2) $\Rightarrow(1)$ is clear, it remains to show that $(4) \Rightarrow(2)$ and $(1) \Rightarrow(3)$. The first implication easily follows by observing that the above action of $\pi_{1}(M)$ on $\mathbb{R}^{n} \times \mathbb{R}^{k}$ is by isometries with respect to the standard metric on $\mathbb{R}^{n+k}$. To prove the second implication, suppose $E$ admits a complete metric with curvature $K \geq 0$ and consider a soul $S$ of $E$. $S$ is nonnegatively curved, homotopy equivalent to $M$, and, thus, flat [1, Corollary 9.5]. Hence, $S$ is diffeomorphic to $M$. If $\rho: \tilde{E} \rightarrow E$ denotes the universal covering, then $\tilde{S}:=\rho^{-1}(S)$ is flat and simply connected (recall that $S \subset E$ is a homotopy equivalence) and, therefore, isometric to $\mathbb{R}^{n}$. Moreover, lines of $\tilde{S}$ are still lines in $\tilde{E}$ by total convexity of $\tilde{S}$, so that by the splitting theorem [1], $\tilde{E}=E_{0} \times \mathbb{R}^{n}$ isometrically. This implies that $E$ splits locally isometrically over $S$. In particular, the Riemannian connection on the normal bundle $\nu$ of $S$ in $E$, induced by the Levi-Civita connection of $E$, is flat.

Corollary. Let $\xi: \mathbb{R}^{2} \rightarrow E \rightarrow M^{n}$ be an oriented vector bundle over a Bieberbach manifold $M$. Then $E$ admits a complete nonnegatively curved metric if and only if the rational Euler class of $\xi$ vanishes.

Proof. If the rational Euler class vanishes, then $\xi$ admits a flat Riemannian connection [2], and the statement follows from (3) of the theorem. Conversely, suppose $E$ admits a complete metric with $K \geq 0$ but $\xi$ has nontrivial rational Euler class. Recall that any Bieberbach $n$-manifold has the torus $T^{n}$ as a finite cover via, say, $f: T^{n} \rightarrow M^{n}$. Then the total space $E^{*}$ of the pullback bundle $f^{*} \xi$ naturally inherits a nonnegatively curved metric. Moreover, the rational, and hence the integral Euler class of $f^{*} \xi$ is nonzero because

$$
f^{*}: H^{*}\left(M^{n} ; \mathbb{Q}\right) \rightarrow H^{*}\left(T^{n} ; \mathbb{Q}\right)
$$

is 1-1. Now, (3) says that $E^{*}$ is diffeomorphic to the total space of a flat plane bundle $\eta$ over $T^{n}$. The rational, and hence the integral Euler class of such a bundle is zero (see, for instance, [5]), so $\eta$ is trivial. Thus, $E^{*}$ is diffeomorphic to $T^{n} \times \mathbb{R}^{2}$. This is impossible, according to:

Proposition. Let $\xi: \mathbb{R}^{k} \rightarrow E \stackrel{\pi}{\longrightarrow} M$ be an oriented vector bundle over a compact, connected, oriented manifold $M$. If $E$ is homeomorphic to a product $E^{\prime} \times \mathbb{R}$, then the (integral) Euler class of $\xi$ vanishes.

Proof. Let $s: M \rightarrow E$ denote the zero section of the bundle. Recall that the Euler class $\chi$ is determined by the following diagram [4]:

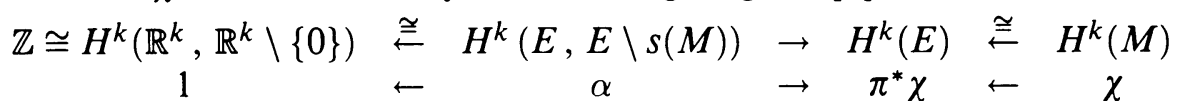

If $h: E \rightarrow E^{\prime} \times \mathbb{R}$ is a homeomorphism, then, by compactness of $M$, there exists $t \in \mathbb{R}$ such that $\phi(M)$ does not intersect $s(M)$, where $\phi$ is the composition

$$
M \stackrel{s}{\longrightarrow} E \stackrel{h}{\longrightarrow} E^{\prime} \times \mathbb{R} \longrightarrow E^{\prime} \longrightarrow E^{\prime} \times\{t\} \hookrightarrow E^{\prime} \times \mathbb{R} \stackrel{h^{-1}}{\longrightarrow} E .
$$

Moreover, $\pi_{*} \phi_{*}[M]= \pm[M]$ since $\phi$ and $\pi$ are homotopy equivalences. By the naturality of the cap product,

$$
0=\alpha \cap \phi_{*}[M]=\pi^{*} \chi \cap \phi_{*}[M] \mapsto \pm \chi \cap[M],
$$

because $\phi(M) \subset E \backslash s(M)$. By Poincaré duality, $\chi=0$. 
Remark. When the rank of the bundle $\xi$ equals the dimension of the base $M$ in the proposition above, the Euler class $\chi$ vanishes if and only if $E$ is homeomorphic to a product $E^{\prime} \times \mathbb{R}$ (thus, it is only the topology of the total space, rather than the bundle structure, which determines the vanishing of $\chi$ ). This follows because the Euler class is the only obstruction to the existence of a nowhere-zero section when $\operatorname{dim} M=k$. In general, it is the primary obstruction.

Example. Recall that for compact, oriented $M^{n}$, every $\alpha \in H^{2}(M)$ determines a unique equivalence class of oriented plane bundles over $M$ with Euler class $\alpha$. By the corollary, among all oriented plane bundles over the $n$-torus, only the total space of the trivial one admits complete metrics of nonnegative curvature. On the other hand, consider a plane bundle over a Bieberbach manifold $M$ whose Euler class is a nonzero torsion element (see [6, Chapter 3] for examples of three-dimensional $M$ with torsion in second cohomology). The total space of such a bundle is not homeomorphic to $M \times \mathbb{R}^{2}$, but, nevertheless, it admits a complete metric with $K \geq 0$.

\section{REFERENCES}

1. J. Cheeger and D. Gromoll, On the structure of complete manifolds of nonnegative curvature, Ann. of Math. (2) 96 (1972), 413-443.

2. F. Kamber and $\mathrm{Ph}$. Tondeur, Flat bundles and characteristic classes of group-representations, Amer. J. Math. 89 (1967), 857-886.

3. J. Milnor, On the existence of a connection with curvature 0, Comment. Math. Helv. 32 (1958), 215-223.

4. J. Milnor and D. Stasheff, Characteristic classes, Princeton Univ. Press, Princeton, NJ, 1974.

5. W. Poor, Differential geometric structures, McGraw-Hill, New York, 1981.

6. J. Wolf, Spaces of constant curvature, Publish or Perish, Cambridge, MA, 1984.

Department of Mathematics, University of Oklahoma, Norman, Oklahoma 73019

E-mail address, M. Özaydin: mozaydin@nsfuvax .math .uoknor . edu

E-mail address, G. Walschap: gwalschap@nsfuvax.math.uoknor.edu 